\title{
Systematic Conservation Planning: an Updated Protocol
}

\author{
Sahotra Sarkar ${ }^{1, *} \&$ Patricia Illoldi-Rangel ${ }^{2}$
}

${ }^{1}$ Department of Philosophy and Section of Integrative Biology, University of Texas, 78712, Austin, TX, USA

${ }^{2}$ Section of Integrative Biology, University of Texas, 78712, Austin, TX, USA

\begin{abstract}
Integrative habitat management requires adaptive management of landscapes and seascapes for productivity (including farming and mining), nature protection, and (human) habitation and recreation. Systematic conservation planning (SCP) is a framework that has been developed during the last decade for a structured approached to prioritizing and managing habitats deemed important for the protection of natural values. Drawing on protocols developed by several groups during the last decade, this paper presents an updated protocol for SCP. The new protocol emphasizes the interactions between components and revision and reiteration of planning stages due to feedback between the components. It also explicitly notes the cultural values and normative assumptions that are built into this framework and emphasizes that adequate planning processes must address the ethical issues that are raised. The paper concludes by noting some of the lacunae of this protocol and difficulties that will be encountered in future extensions to satisfy all goals of integrative habitat management.
\end{abstract}

Key words: Conservation Area Networks, Decision Support, Environmental Ethics, Reserve Design, Reserve Selection, Systematic Conservation Planning.

\section{Introduction}

In almost all circumstances, biodiversity conservation is not the only ethically salient use of a landscape or seascape. Other potential such uses include human habitation and recreation as well as production (including agriculture, pisciculture, and mineral resource extraction). Human interests are particularly important when areas prioritized by conservationists for natural values are also linked to the well-being of economically deprived groups, as is often the case in the biologically most important areas of the world.

The incorporation of these often-divergent goals into a coherent conceptual and normative framework is the goal of integrative habitat management (Sarkar \& Montoya 2010). Meanwhile, the less ambitious program of systematic conservation planning (SCP) constitutes a framework that has been developed for the limited goal of biodiversity conservation and, when possible, the incorporation of other natural values and some socio-political goals through multi-criteria analysis (MCA) (Margules \& Pressey 2000, Margules \& Sarkar 2007).

\footnotetext{
*Send correspondence to: Sahotra Sarkar

Department of Philosophy and Section of Integrative Biology,

University of Texas, 78712, Austin, TX, USA

E-mail: sarkar@mail.utexas.edu
}

SCP is a structured multi-component stage-wise approach to identifying conservation areas and devising management policy, with feedback, revision, and reiteration, where needed, at any stage (Margules \& Sarkar 2007). The areas prioritized by such an approach form a Conservation Area Network (CAN). Conservation areas must be distinguished from traditional reserves because SCP envisions the use of a wide variety of habitat management strategies including, but not limited to, the creation of national parks, biosphere reserves, and conservation easement practices (Sarkar 2003). Selecting CANs is relatively easy thanks to 25 years of research in this area; implementing them and devising adequate management and monitoring strategies remain difficult reasearch problems within contemparly SCP.

A critical aspect of such planning has been the development and extensive use of decision support software tools incorporating algorithms specifically designed for the solution of planning problems. These tools include ConsNet (Ciarleglio et al. 2009, 2010), C-Plan (Pressey et al. 2009), LQGraph (Fuller \& Sarkar 2006), Marxan (Ball et al. 2009), MultCSync (Moffett et al. 2005), ResNet (Sarkar et al. 2002, 2009), and Zonation (Moilanen et al. 2009a), and have recently been reviewed by Sarkar et al. (2006) and Moilanen et al. (2009b). The development of algorithms and software tools for planning decision support goes back to the 1980s 
(Justus \& Sarkar 2002, Pressey 2002) and became a major research component within conservation biology; it was one of the features that distinguished that newly-emerging field from traditional ecology (Sarkar 2004).

The stages of SCP are shown in Figure 1 which generalizes the work of several researchers, primarily from 2000 to 2007 (Cowling \& Pressey 2003, Groves 2003, Margules \& Pressey 2000, Margules \& Sarkar 2007, Sarkar 2004, 2005). The central goals of SCP are (Margules \& Sarkar 2007):

- adequate representation of all components of biodiversity in conservation area networks (CANs);

- ensuring the persistence of biodiversity into the future;

- achieving these ends with as much economy of resources as possible.
The first two of these were articulated by Margules \& Pressey (2000) in a highly influential paper in Nature in 2000; the third was added by Margules \& Sarkar (2007) which should be consulted for more detail. Economy was included in this set of fundamental objectives of SCP because resources for biodiversity conservation are never unlimited and should be optimally allocated. In particular, because such resources could potentially be used to promote human well-being, efficient resource allocation is an ethical imperative.

\section{The Modified SCP Framework and Updated Protocol}

The first explicit framework for SCP was developed by Margules \& Pressey (2000). Subsequently, in an important contribution, Cowling \& Pressey (2003) noted that the identification of appropriate stakeholders should be regarded as a distinct component. Sarkar $(2004,2005)$ introduced

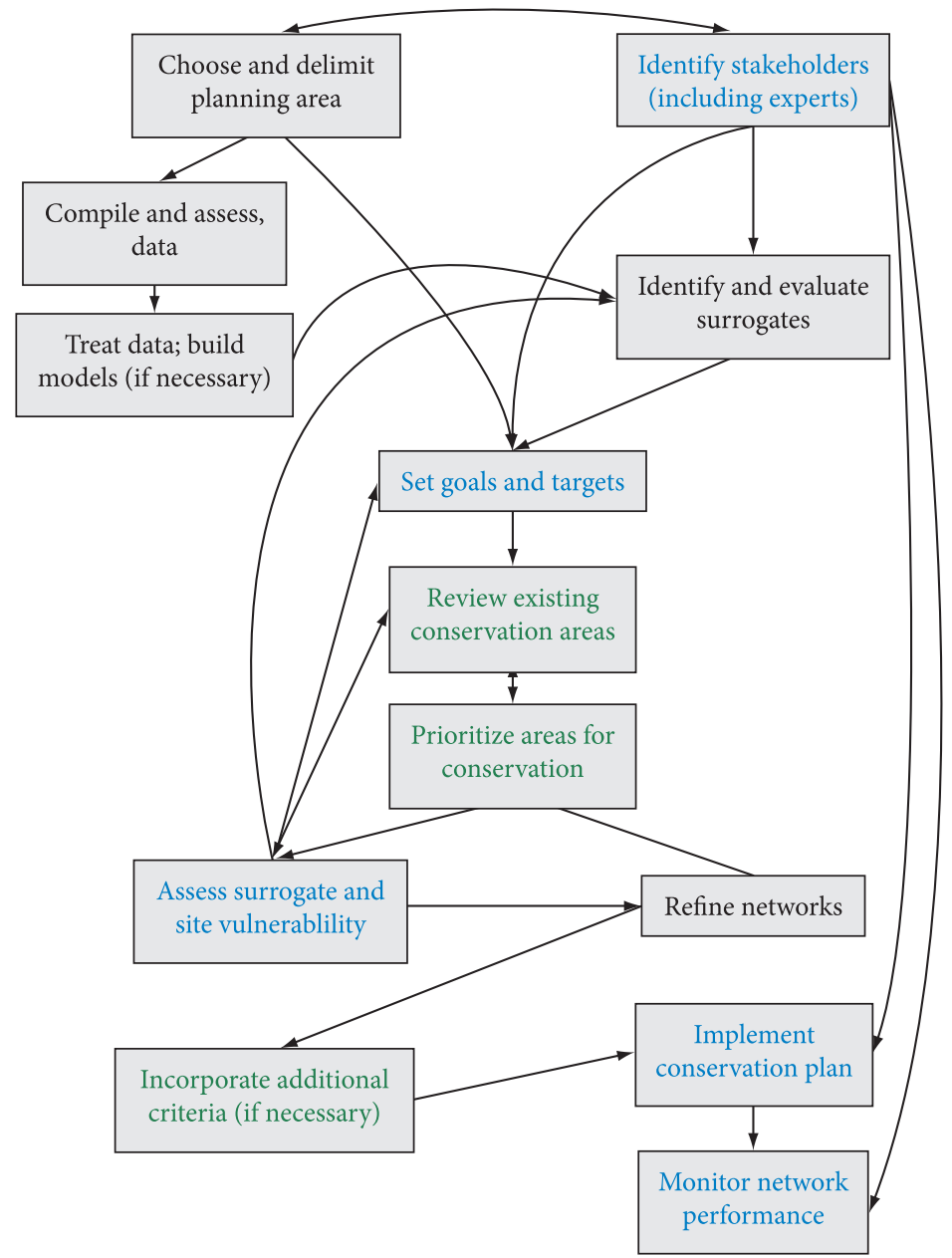

Figure 1. Stages of Systematic Conservation Planning. Arrows indicate which components directly influence which others. A bidirectional arrow indicates feedback. Only the major interactions between the components are shown. There is potential for feedback between almost any two components of this framework. Boxes with text in green indicate aspects that are well-understood, those with text in black are aspects which are fairly well-understood, and those with blue text are areas that remain poorly understood and subject to much ongoing research. 
viability assessment as an explicit part of the protocol; it had been implicitly assumed by Margules \& Pressey's (2000) earlier discussion. He also introduced formal multi-criteria analysis (MCA) into the framework. Figure 1 introduces two sets of innovations from the discussion in Margules \& Sarkar (2007) which had attemptetd to synthesize all earlier developments:

a. Two new components have been added:

i. The identification of the planning unit (study area) is explicitly introduced as a separate component. The importance of having such a component emerged from recent work in developing a conservation and economic development plan in Papua (Indonesian New Guinea) by Conservation International and University of Texas personnel during the course of which it became clear that different stakeholders did not have identical views on how the planning unit should be delimited (Conservation International 2010);

ii. A separate component for building (biological and socio-economic) models has been added. The experience of a large number of recent planning exercises has indicated that this is a major part of the planning process (Pawar et al. 2007, Illoldi-Rangel et al. 2008, Sarkar et al. 2009, Zafra-Calvo et al. 2010). In such a context, treating modeling and data treatment as part of data compilation (Margules \& Pressey 2000, Margules \& Sarkar 2007) no longer seems appropriate.

b. While Margules \& Sarkar (2007) explicitly noted that the components of SCP interacted with each other, they did not indicate which interactions are most important. The arrows in Figure 1 remove that lacuna, with the most interesting ones being the bidirectional arrows which indicate the possibility of significant feedback which means that there may be extensive revision and reiteration of those components. The arrows are intended only to capture the most important interactions, those that planners should be cognizant of in every planning exercise. Contextual interactions will typically introduce many other links (Sarkar \& Montoya 2010).

There are thirteen components, also called stages, of SCP. As Figure 1 emphasizes, these stages should not be viewed as a linear sequence-thus, "stages" is a bit misleading but has become standard termionology (Margules \& Sarkar 2007) - though some components depend on the completion of other (ipso facto earlier) ones. Because of this, the description of many of the components below must necessarily refer to components only described later:

\section{Choose and delimit the planning region}

The precise geographical boundaries of the planning region should be explicitly discussed at the beginning of the planning process. There should be explicit discussion on how the boundaries should be drawn, for instance, whether they should be based on ecological or political criteria, and what criteria should be used. Different stakeholders may have different preferences for the boundaries of the planning unit. Thus the choice of the planning region interacts with the identity and views of the stakeholders. The boundaries of the planning region may have a strong influence on what types of data compilation and analysis may be feasible (and, hence, the interaction with the data compilation component of SCP in Figure 1). Moreover, different components of the planning process may require different study areas. For instance, if the planning is defined using political criteria, species distribution models (see Stage 4 below) may still have to be constructed for a larger area determined by ecological boundaries. (Otherwise, ecological models are likely to be unreliable.)

\section{Identify all stakeholders (Cowling \& Pressey 2003)}

Conservation plans have little chance of successful implementation if they do not manage to negotiate socio-political issues in the planning region, incorporate the constraints, and take advantage of opportunities. Prospects for successful implementation are enhanced if all the relevant agents participate in the planning process from the beginning. The stakeholders will also have a role in implementing and monitoring a conservation plan at later stages (see below). Stakeholders include biological and other experts. Obviously, the delineation of a planning region depends on the expert stakeholders and the choice of the planning region influences who qualifies as experts and other stakeholders. There is feedback between these two early stages.

Additionally, it should be noted that although stakeholders may disagree with one another, area prioritization methods (see below) typically presuppose that there is a single decision-maker. This is equivalent to requiring a consensus among stakeholders, but recent work on group decisions on conservation planning indicates that this may be highly problematic (Frank \& Sarkar 2010).

Moreover, by and large, SCP has completely ignored the critical normative question that should be addressed before any plan is developed (Sarkar \& Montoya 2010): who is a legitimate stakeholder? While space constraints will not permit a detailed discussion here of this crucial question of conservation ethics, it should be emphasized that it is far from clear that external agents (including conservation "experts" and non-governmental organization [NGOs]) are legitimate stakeholders. Perhaps the only legitimate role for these agents is of decision support without participation in 
decision-making. The fact that large conservation NGOs and trans-national organizations have been responsible for serious disenfranchisement and harm to local groups should also always be kept in mind (Guha 1989, 1997, Sarkar 1998, 1999, Dowie 2009). The ethical issue broached here is independent of the prudential question whether appropriate takeholder have been identified so that any plan can be implemented.

\section{Compile and assess data}

Conservation planning requires both biological and socio-political data. There is almost never sufficient resources to collect all the data that would be useful for planning purposes. Data collection, for instance through surveys, should be cost-efficient and focused on those parameters that are the most important. These are determined by the study region and the planning goals and objectives. In the past, SCP has been criticized for requiring data that cannot be obtained in practice (Redford et al. 1997); however, it is clear by now that there is no terrestrial region for which there is not enough data to act as adquate estimator-surrogates for biodiversity (Sarkar et al. 2006) and this criticism is no longer relevant. Three developments have contributed to this improved situations: i) the creation of many regional and international databases by digitizing existing data [for instance, the Global Biodiversity Inventory Facility (GBIF)]; ii) better modeling and interpolation techniques, as will be noted below (Hijmans et al. 2005, Elith et al. 2006); and iii) the collection and dissemination of a large body of remote-sensed data including high resolution digital elevation maps (United Stations Geological Survey 1998).

\section{Treat data and construct models as necessary}

Almost all data have inbuilt spatial and other biases that have to be removed through statistical refinement and modeling. For biological data (especially distributions of taxa) this is true because most available data were not collected through carefully-designed surveys but opportunisitically, in response to the needs of individual (often taxonomic) research projects. For physical environmental data biases may arise due to the location of collection points which are often close to socially relevant localities such as urban centers. Interpolation of such data, especially climatic data, to increasingly finer resolutions is now standard (Hijmans et al. 2005).

In many contexts of biodiversity conservation, where species are taken as estimator-surrogates (see Stage 5 below), species' geographical distributions typically have to be modeled from sparse opportunistic records and modeled environmental data. Species distribution modeling is increasingly being used for this purpose (Elith et al. 2006) especially given the wide of recently introduced maximum entropy-based predictive algorithms (Moilanen et al. 2009b, Phillips \& Dudik 2008).

\section{Identify and evaluate biodiversity surrogates}

Care must be taken to ensure that biodiversity is appropriately represented and quantified. First, appropriate "true surrogates" for biodiversity (Sarkar 2002, Sarkar \& Margules 2002), also called "biodiversity constituents" (Sarkar 2008) must be selected by stakeholders to represent the most important components of the regional biota that deserve conservation attention. Biodiversity constituents must be identified because, given ubiquitous resource limitations, not all biota will merit conservation. What these constituents are reflects cultural values and there has been much discussion and disagreement over this topic (Sarkar 2008). For instance, among NGOs, Consevation International uses at- risk and limited-range species whereas The Nature Conservancy uses ecosystem (habitat) types. In the United States, almost all governmental agencies use at-risk species; in the European Union, emphasis is on habitat types. The point is that there is no purely scientific answer to the question and the cultural choices involved must be negotiated by the stakeholders. If full distributional information on the biodiversity consituents is not obtainable, as is typically the case, "estimator-surrogates" with adequate performance must be chosen. This means that it must be quantitatively established that these estimator-surrogates can successfully replace the true surrogates in planning protocols, and methods of surrogacy analysis have been developed for this purpose (Sarkar et al. 2005). For instance, in many regions, environmental features are adequate estimator-surrogates for many taxa that are taken to be biodiversity constituents.

\section{Set explicit biodiversity goals and targets}

It is crucial to be explicit, right from the beginning, about what constitutes adequate biodiversity protection. This prevents many potential later disagreements about the merits of various choices. Quantitative targets of representation CANs must be set for all biodiversity surrogates. Spatial configuration and other goals must similarly be explicitly specified. How to set proper targets and goals is poorly understood. The use of biodiversity representation targets has often been criticized on the ground that they have no firm biological basis while having a significant effect on the plans being developed (Justus et al. 2002). However, a recent review suggests that this criticism is misplaced so long as targets and goals are properly set (Carwardine et al. 2009). Targets should ensure that CANs should be large enough to ensure that, provided the CAN has a proper spatial configuration, biodiversity constituents can persist indefinitely into the future. In practice, the ecological problems posed by this goal can only be solved for a very few species (through population viability analysis) for which adequate long-term data are available. Most of the time targets and goals have to be set on the basis of ecological intuitions of experts which is never an ideal solution (Margules \& Sarkar 2007). 


\section{Review existing conservation areas for performance with respect to targets}

The existing CAN, if there is one, must be analyzed to determine the extent to which it already satisfies the specified goals and targets. Typically, most regions will have an existing set of protected areas though these may contribute little to the goals and targets if they have not been selected through a systematic process (Pressey 1994). This is perhaps the most straightforward stage for SCP.

\section{Prioritize additional areas for conservation management}

New areas must be prioritized so that the specified goals and targets may be met when these areas are included in an expanded CAN for the region. The problem is one of constrained optimization: achieving the representation of all surrogates at least up to their targets (the constraint) in as little area as possible (the opitmization problem) (Sarkar et al. 2006). This stage may involve only achieving representation targets or also incorporate other criteria, both spatial and socio-political, as additional constraints. Ensuring that all biodiversity surrogates meet their specified representation targets in a nominal CAN is the best understood part of the planning process (Margules et al. 1988, Sarkar et al. 2006, Margules \& Sarkar 2007). A wide variety of algorithms have been explored for the CAN selection problem, including exact, heuristic, and metaheuristic algorithms (Sarkar et al. 2006). Many software packages, including all those mentioned in Section 1, have been devised with this problem primarily in mind. Originally the emphasis in the algorithms was on achieving adequate surrogate representation in minimum area; more recently, it has shifted towards also ensuring that criteria related to spatial configuration are satisfied. The use of such algorithmic methods was once subjected to unjustified criticism by The Nature Conservancy on grounds of insufficient data (Redford et al. 1997; for a response, see Justus \& Sarkar 2002) but those criticisms have since been withdrawn (Grooves 2003).

\section{Assess biodiversity constituent and selected area vulnerabilities}

A selected area may itself be vulnerable, in which case there is usually a poor prognosis for all its biodiversity constituents. This vulnerability can arise from socio-politcial factors (for instance, development threat), existing ecological factors, or global change factors (such as climate change). Alternatively, only some of the constituents in the area may be vulnerable because of the quality of the habitat. If those constituents require that area to meet the relevant goals and targets then, the area itself should be considered vulnerable from the perspective of designing an adequate CAN. Assessing the vulnerability of constituents can often be very difficult in practice for the same reasons that setting quantitative targets of representation was deemed difficult earlier. This part of SCP remains very poorly understood (Margules \& Sarkar 2007).

\section{Refine the network of selected areas}

If vulnerable areas are not entirely irreplaceable (Pressey 1999), a good strategy is to exclude them from nominal CANs and repeat the CAN selection process to prioritize new areas in order to ensure that all goals and targets are met in a new nominal CAN. The vulnerability analysis must then be performed again, and the entire cycle must be reiterated until all goals and targets are met in a "safe" set of nominal conservation areas. Since the techniques used are that of CAN selection, this has become a relatively straightforward process.

\section{Carry out multi-criteria analysis (MCA)}

If not all relevant criteria were incorporated at the stage of prioritizing new areas (Stage 8), a MCA must be performed to ensure that these criteria are incorporated into the design. These criteria fall into two main categories: i) those involving the spatial configuration of CANs-size, shape, connectivity, replication, dispersion, and alignment (Sarkar et al. 2006); and ii) those incorporating socio-political imperatives-economic cost, social cost, recreational value, cultural role, educational role, etc (Moffett \& Sarkar 2006). The latter include ethical imperatives underlying policy preferences. Typically MCA is done by generating a large number of "solutions" or nominal CANs each of which satisfies the criteria that were used for area prioritization. These solutions are now evaluated and ranked using the other criteria. While a large number of MCA techniques have been used within SCP (Moffett \& Sarkar 2006), many are known to be ad hoc in the sense of not being consistent with standard decision theory and economic analyses (Dyer 1990, Moffett et al. 2005). However, methods of MCA geared towards SCP that are fully consistent with decision theory have been implemented in several recent software decision tools (Moffett et al. 2005, Ciarleglio et al. 2009, 2010). By now the use of MCS in SCP is fairly well-understood.

\section{Implement conservation plan}

A nominal CAN must be implemented by devising appropriate management plans and then ensuring that they are put into practice. Implementation plans must take all relevant contextual issues into account. Management options may well include continued traditional use; they are certainly not restricted to the conventional Northern strategy of human exclusionn (Sarkar \& Montoya 2010). What strategy is most adequate is an empirical question, not appropriately decided on ideological grounds (Sarkar 1999, 2005). While scientific analyses contribute towards devising a plan, implementation is largely a socio-political 
process, which is poorly understood. In particular, plans may only be incompletely implemented, or implemented part by part over a period of years. These possibilities change the nature of the (formal) CAN selection problem and pose considerable computational challenges. While these issues have been long recognized within SCP (Sarkar et al. 2006), solutions have not been forthcoming. Meeting the socio-political challenges of making conservation work in the field has been even more difficult with very few exceptions (Conservation International 2010). It is expected that having an appropriate set of stakeholders from the beginning would help this process but, so far, there is little to report from the field. (Thus, the earliest stage of the SCP process is linked with a relatively late stage.)

\section{Monitor network performance}

Biodiversity conservation is not a one-off process of delineating CANs which can then be left to persist on their own. Both human encroachments and natural changes can alter the conservation status of a delineated area. Global factors such as climate change also play a role. Consequently, CAN performance must be continually monitored, and the planning process must be periodically repeated as part of adaptive management. This is easy to say but has proved hard to achieve. Very little research has been devoted to devising appropriate monitoring protocols even when attention is restricted to the ecology of a CAN. However, socio-political factors must also be incorporated into the process and this will require the attention of appropriate stakeholders. (Thus, once again the earliest stage of the SCP process is linked with a late stage.)

\section{Concluding Remarks}

As noted earlier (in Section 1), biodiversity conservation is not the only legitimate use of habitats. Recently, Sarkar \& Montoya (2010) have made an initial pass at developing a protocol for integrative habitat planning (IHP) by extension and modification of the framework described here. It became clear from that analysis that any IHP protocol would be much more contextual and also depend very criticallly on the socio-political goals of the planners. Sarkar \& Montoya's (2010) version assumed the goals of political ecology with its emphasis on local control, participatory democracy, and distributive justice. But other political goals are clearly possible. Developing a wide consensus on what should be assumed in such IHP protocols, beyond the comparatively uncontroversial assumptions of SCP, remains an important task for the future.

The structure depicted in Figure 1 only incorporates the most important interactions between the components of SCP. Moreover, it is probably not unique in the sense that other structures of the interaction network may implement the same genearal ideas. The point is that any such protocol should not be used mechanically. Rather it should serve as a guide: some components may not be necessary-for instance, the identity of the stakeholders may be fully specified and not subject to change; many features may well need modification to reflect context. Figure 1 should be used flexibly.

Finally, for the future success of SCP, attention should be directed to those components that are at present poorly understood. There are five of these: identification of stakeholders, setting goals and targets, vulnerability assessment, implementation, and monitoring. However, additional research on all other components of Figure 1 is desirable, especially those in black text because, though they remain partly understood, there remains ample scope for improvement.

\section{Acknowledgements}

For years of collaboration and discussion, both authors would like to thank Chris Margules and Victor Sánchez-Cordero. Thanks are also due to Anshu Aggarwal, Francisco Botello, Michael Ciarleglio, Trevon Fuller, Justin Garson, Chris Kelley, Miguel Linaje, Vanessa Lujan, Alex Moffett, and Chris Pappas for input in the development of this protocol.

\section{References}

Ball IR, Possingham HP \& Watts ME, 2009. Marxan \& relatives: Software for spatial conservation prioritization. In Moilanen A, Wilson KA \& Possingham HP (eds.). Spatial Conservation Prioritization: Quantitative Methods and Computational Tools. Oxford: Oxford University Press. p. 185-195.

Carwardine J et al., 2009. Hitting the target and missing the point: target-based conservation planning in context. Conserv Lett, 2:4-11.

Ciarleglio M et al., 2009. ConsNet: New software for the selection of conservation area networks with spatial and multi-criteria analyses. Ecography, 32:205-209.

Ciarleglio M et al., 2010. ConsNet-a tabu search approach to the spatially coherent conservation area network design problem. Journal Heuristics, in press.

Conservation International, 2010. Optimizing conservation and production: a collaboration between conservation and business. Report, Jakarta: Conservation International.

Cowling RM \& Pressey RL, 2003. Introduction to systematic conservation planning in the Cape Floristic Region. Biological Conservation, 112:1-13.

Dowie M, 2009. Conservation Refugees: The Hundred-Year Conflict between Global Conservation and Native Peoples. Cambridge: MIT Press.

Dyer J, 1990. Remarks on the Analytic Hierarchy Process. Management Science, 36:249-258.

Elith J et al., 2006. Novel methods improve prediction of species' distributions from occurrence data. Ecography, 29:129-151.

Frank DM \& Sarkar S, 2010. Group decisions in biodiversity conservation: Implications from game theory. PLoS ONE, in press. 
Fuller T \& Sarkar S, 2006. LQGraph: A software package for optimizing connectivity in conservation planning. Environmental Modelling \& Software, 21:750-755.

Groves C, 2003. Drafting a Conservation Blueprint: A Practitioner's Guide to Planning for Biodiversity. Washington, DC: Island Press.

Guha R, 1989. Radical American environmentalism and wilderness preservation: A third world critique. Environmental Ethics, 11:71-83.

Guha R, 1997. The authoritarian biologist and the arrogance of anti-humanism: Wildlife conservation in the Third World. Ecologist, 27:14-20.

Hijmans R et al., 2005. Very high resolution interpolated climate surfaces for global land areas. International Journal of Climatology, 25:1965-1978.

Illoldi-Rangel P et al., 2008. Solving the maximum representation problem to prioritize areas for the conservation of terrestrial mammals at risk in Oaxaca. Divers Distribuition, 14:493-508.

Justus J et al., 2008. Influence of representation targets on the total area of conservation area networks. Conservation Biology, 22:673-682.

Justus J \& Sarkar S, 2002. The principle of complementarity in the design of reserve networks to conserve biodiversity: A preliminary history. Journal of Biosciences, 27:421-435.

Margules CR et al., 1988. Selecting networks of reserves to maximize biological diversity. Biological Conservation, 43:63-76.

Margules CR \& Pressey RL, 2000. Systematic conservation planning. Nature, 405:249-253.

Margules CR \& Sarkar S, 2007. Systematic Conservation Planning. Cambridge: Cambridge University Press.

Moffett A et al., 2006. Integrating biodiversity representation with multiple criteria in North-Central Namibia using non-dominated alternatives and a modified Analytic Hierarchy Process. Biological Conservation, 129:181-191.

Moffett A et al., 2005. MultCSync: A software package for incorporating multiple criteria in conservation planning. Environmental Modelling \& Software, 20: 1315-1322.

Moffett A \& Sarkar S, 2006. Incorporating multiple criteria into the design of conservation area networks: A minireview with recommendations. Divers Distribuition, 12:125-137.

Moilanen A, Kujala H \& Leathwick JR, 2009a. The Zonation framework and software for conservation prioritization. In Moilanen A, Wilson KA \& Possingham HP (eds.). Spatial Conservation Prioritization: Quantitative Methods and Computational Tools. Oxford: Oxford University Press. p. 196-210.

Moilanen A, Wilson KA \& Possingham HP (eds.), 2009b. Spatial Conservation Prioritization: Quantitative Methods and Computational Tools. Oxford: Oxford University Press.

Pawar S et al., 2007. Conservation assessment and prioritization of areas in Northeast India: priorites for amphibians and reptiles. Biological Conservation, 136: 346-361.
Phillips SJ \& Dudik M, 2008. Modeling of species distributions with Maxent: new extensions and a comprehensive evaluation. Ecography, 31:161-175.

Pressey RL, 1994. Ad Hoc reservations: Forward of backward steps in developing representative reserve systems. Conservation Biology, 8:662-668.

Pressey RL, 1999. Applications of irreplaceability analysis to planning and management problems. Parks, 9:42-51.

Pressey RL, 2002. The first reserve selection algorithm: A retrospective on Jamie Kirkpatrick's 1983 paper. Progress in Physical Geography, 26:434-441.

Pressey RL et al., 2009. The C-Plan conservation planning system: Origins, applications, and possible futures. In Moilanen A, Wilson KA \& Possingham HP (eds.). Spatial Conservation Prioritization: Quantitative Methods and Computational Tools. Oxford: Oxford University Press. p. 211-234.

Redford K et al., 1997. Designing a Geography of Hope: Guidelines for Ecoregion Conservation in The Nature Conservancy. Arlington: The Nature Conservancy.

Sarkar S, 1998. Restoring wilderness or reclaiming forests? Terra Nova, 3:35-52.

Sarkar S, 1999. Wilderness preservation and biodiversity conservation keeping divergent goals distinct. BioScience, 49:405-412.

Sarkar S, 2002. Defining 'biodiversity'; assessing biodiversity. The Monist, 85:131-155.

Sarkar S, 2003. Conservation area networks. Conservation Society, 1: v-vii.

Sarkar S, 2004. Conservation biology. In Zalta EN (ed.). The Stanford Encyclopedia of Philosophy. Stanford: Stanford University. Available from: plato.stanford.edu/archives/ sum2004/entries/conservation-biology/.

Sarkar S, 2005. Biodiversity and Environmental Philosophy: An Introduction to the Issues. Cambridge: Cambridge University Press.

Sarkar S, 2008. Norms and the conservation of biodiversity. Resonance, 13:627-637.

Sarkar S et al., 2002. Place prioritization for biodiveristy content. Journal Biosciences, 27:339-346.

Sarkar S et al., 2009. The ConsNet software platform for systematic conservation planning. In Moilanen A, Wilson KA \& Possingham HP (eds.). Spatial Conservation Prioritization: Quantitative Methods and Computational Tools. Oxford: Oxford University Press. p. 235-248.

Sarkar S et al., 2005. Effectiveness of environmental surrogates for the selection of conservation area networks. Conservation Biology, 19:815-825.

Sarkar S \& Margules CR, 2002. Operationalizing biodiversity for conservation planning. Journal Biosciences, 27:299-308.

Sarkar S \& Montoya M., 2010. Beyond parks and reserves: The ethics and politics of conservation with a case study from Perú. Biological Conservation, in press. 
Sarkar S et al., 2006. Biodiversity conservation planning tools: Present status and challenges for the future. Annual Review of Environment and Resources, 31: 123-159.

Sarkar S et al., 2009. Systematic conservation assessment for the Mesoamerica, Chocó and Tropical Andes biodiversity hotspots: A preliminary analysis. Biodivers Conservation, 18:1793-1828.

United Stations Geological Survey, 1998. GTOPO Global 30 arc-second digital elevation model. Available from: http:// eros.usgs.gov/products/elevation/gtopo30/hydro
Zafra-Calvo N et al., 2010. Prioritizing areas for conservation and vegetation restoration in post-agricultural landscapes: A Biosphere Reserve plan for Bioko, Equatorial Guinea. Biological Conservation, 143:787-794.

Received: May 2010

First Decision: June 2010

Accepted: June 2010 